\title{
Observation of Fine Time Structures in the Cosmic Proton and Helium Fluxes with the Alpha Magnetic Spectrometer on the International Space Station
}

M. Aguilar, ${ }^{27}$ L. Ali Cavasonza, ${ }^{1}$ B. Alpat, ${ }^{32}$ G. Ambrosi, ${ }^{32}$ L. Arruda, ${ }^{25}$ N. Attig, ${ }^{22}$ S. Aupetit, ${ }^{18}$ P. Azzarello, ${ }^{17}$ A. Bachlechner, ${ }^{1}$ F. Barao, ${ }^{25}$ A. Barrau, ${ }^{18}$ L. Barrin,${ }^{16}$ A. Bartoloni, ${ }^{37}$ L. Basara, ${ }^{35}$ S. Başeğmez-du Pree,${ }^{6}$ M. Battarbee ${ }^{45}$ R. Battiston, ${ }^{35,36, a}$ U. Becker, ${ }^{10}$ M. Behlmann, ${ }^{10}$ B. Beischer, ${ }^{1}$ J. Berdugo, ${ }^{27}$ B. Bertucci, ${ }^{32,33}$ K. F. Bindel, ${ }^{23}$ V. Bindi, ${ }^{20}$ W. de Boer, ${ }^{23}$ K. Bollweg, ${ }^{21}$ V. Bonnivard, ${ }^{18}$ B. Borgia, ${ }^{37,38}$ M. J. Boschini, ${ }^{29}$ M. Bourquin, ${ }^{17}$ E. F. Bueno, ${ }^{39}$ J. Burger, ${ }^{10}$ F. Cadoux,${ }^{17}$ X. D. Cai,${ }^{10}$ M. Capell, ${ }^{10}$ S. Caroff, ${ }^{3}$ J. Casaus, ${ }^{27}$ G. Castellini,${ }^{15}$ F. Cervelli, ${ }^{34}$ M. J. Chae,${ }^{40}$ Y. H. Chang, ${ }^{11}$ A. I. Chen, ${ }^{10}$ G. M. Chen, ${ }^{6}$ H. S. Chen,${ }^{6,7}$ Y. Chen, ${ }^{17}$ L. Cheng, ${ }^{41}$ H. Y. Chou, ${ }^{11}$ E. Choumilov, ${ }^{10}$ V. Choutko, ${ }^{10}$ C. H. Chung, ${ }^{1}$ C. Clark, ${ }^{21}$ R. Clavero, ${ }^{24}$ G. Coignet, ${ }^{3}$ C. Consolandi, ${ }^{20}$ A. Contin,${ }^{8,9}$ C. Corti, ${ }^{20}$ W. Creus, ${ }^{44}$ M. Crispoltoni,,${ }^{32,33}$ Z. Cui, ${ }^{41}$ K. Dadzie, ${ }^{10}$ Y. M. Dai, ${ }^{5}$ A. Datta, ${ }^{20}$ C. Delgado, ${ }^{27}$ S. Della Torre, ${ }^{29}$ M. B. Demirköz,${ }^{2}$ L. Derome, ${ }^{18}$ S. Di Falco, ${ }^{34}$ F. Dimiccoli, ${ }^{35,36}$ C. Díaz, ${ }^{27}$ P. von Doetinchem, ${ }^{20}$ F. Dong, ${ }^{31}$ F. Donnini, ${ }^{32,33}$ M. Duranti, ${ }^{32}$ D. D’Urso, ${ }^{32, b}$ A. Egorov, ${ }^{10}$ A. Eline,${ }^{10}$ T. Eronen ${ }^{45}$ J. Feng, ${ }^{10}$ E. Fiandrini, ${ }^{32,33}$ P. Fisher ${ }^{10}$ V. Formato,${ }^{32}$ Y. Galaktionov, ${ }^{10}$ G. Gallucci, ${ }^{34}$ R. J. García-López, ${ }^{24}$ C. Gargiulo, ${ }^{16}$ H. Gast, ${ }^{1}$ I. Gebauer, ${ }^{23}$ M. Gervasi, ${ }^{29,30}$ A. Ghelfi, ${ }^{18}$ F. Giovacchini, ${ }^{27}$ D. M. Gómez-Coral, ${ }^{28}$ J. Gong, ${ }^{31}$ C. Goy, ${ }^{3}$ V. Grabski, ${ }^{28}$ D. Grandi, ${ }^{29}$ M. Graziani, ${ }^{23}$ K. H. Guo, ${ }^{19}$ S. Haino, ${ }^{44}$ K. C. Han,${ }^{26}$ Z. H. He, ${ }^{19}$ M. Heil,${ }^{10}$ J. Hoffman, ${ }^{20}$ T. H. Hsieh, ${ }^{10}$ H. Huang, ${ }^{44, c}$ Z. C. Huang, ${ }^{19}$ C. Huh, ${ }^{14}$ M. Incagli, ${ }^{34}$ M. Ionica, ${ }^{32}$ W. Y. Jang, ${ }^{14}$ Yi Jia, ${ }^{10}$ H. Jinchi, ${ }^{26}$ S. C. Kang, ${ }^{14}$ K. Kanishev,${ }^{35,16}$ B. Khiali, ${ }^{11}$ G. N. Kim, ${ }^{14}$ K. S. Kim,${ }^{14}$ Th. Kirn, ${ }^{1}$ C. Konak, ${ }^{2}$ O. Kounina, ${ }_{10}$ A. Kounine, ${ }^{10}$ V. Koutsenko, ${ }^{10}$ A. Kulemzin, ${ }^{10}$ G. La Vacca, ${ }^{29,30}$ E. Laudi, ${ }^{16}$ G. Laurenti, ${ }^{8}$ I. Lazzizzera, ${ }^{35,36}$ A. Lebedev, ${ }^{10}$ H. T. Lee, ${ }^{43}$ S. C. Lee, ${ }^{44}$ C. Leluc, ${ }^{17}$ H. S. Li ${ }^{42}$ J. Q. Li, ${ }^{31}$ Q. Li,${ }^{31}$ T. X. Li, ${ }^{19}$ Z. H. Li, ${ }^{6}$ Z. Y. Li,${ }^{44, d}$ C. Light, ${ }^{20}$ S. Lim, ${ }^{14}$ C. H. Lin,${ }^{44}$ P. Lipari, ${ }^{37}$ T. Lippert, ${ }^{22}$ D. Liu, ${ }^{11}$ Hu Liu, ${ }^{10,}$ V. D. Lordello, ${ }^{39}$ S. Q. Lu, ${ }^{44, d}$ Y. S. Lu, ${ }^{6}$ K. Luebelsmeyer, ${ }^{1}$ F. Luo, ${ }^{41}$ J. Z. Luo, ${ }^{31}$ X. Luo, ${ }^{20}$ S. S. Lyu, ${ }^{19}$ F. Machate, ${ }^{1}$ C. Mañá, ${ }^{27}$ J. Marín, ${ }^{27}$ T. Martin, ${ }^{21}$ G. Martínez,${ }^{27}$ N. Masi, ${ }^{8}$ D. Maurin, ${ }^{18}$ A. Menchaca-Rocha, ${ }^{28}$ Q. Meng, ${ }^{31}$ V. M. Mikuni, ${ }^{39}$ D. C. Mo, ${ }^{19}$ P. Mott, ${ }^{21}$ T. Nelson, ${ }^{20}$ J. Q. Ni, ${ }^{19}$ N. Nikonov, ${ }^{1}$ F. Nozzoli, ${ }^{32, f}$ A. Oliva, ${ }^{27}$ M. Orcinha,${ }^{25}$ M. Palermo, ${ }^{20}$ F. Palmonari, ${ }^{8,9}$ C. Palomares, ${ }^{27}$ M. Paniccia, ${ }^{17}$ M. Pauluzzi, ${ }^{32,33}$ S. Pensotti ${ }^{29,30}$ C. Perrina,${ }^{17}$ H. D. Phan,${ }^{10}$ N. Picot-Clemente, ${ }^{13}$ F. Pilo, ${ }^{34}$ C. Pizzolotto, ${ }^{32, g}$ V. Plyaskin, ${ }^{10}$ M. Pohl, ${ }^{17}$ V. Poireau, ${ }^{3}$ A. Popkow, ${ }^{20}$ L. Quadrani,${ }^{89}$ X. M. Qi ${ }^{19}$ X. Qin, ${ }^{10}$ Z. Y. Qu, ${ }^{44, h}$ T. Räihä, ${ }^{1}$ P. G. Rancoita, ${ }^{29}$ D. Rapin, ${ }^{17}$ J. S. Ricol, ${ }^{18}$ S. Rosier-Lees, ${ }^{3}$ A. Rozhkov,${ }^{10}$ D. Rozza, ${ }^{29,30}$ R. Sagdeev, ${ }^{12}$ S. Schael, ${ }^{1}$ S. M. Schmidt, ${ }^{22}$ A. Schulz von Dratzig, ${ }^{1}$ G. Schwering, ${ }^{1}$ E. S. Seo, ${ }^{13}$ B. S. Shan, ${ }^{4}$ J. Y. Shi, ${ }^{31}$ T. Siedenburg, ${ }^{1}$ D. Son, ${ }^{14}$ J. W. Song, ${ }^{41}$ M. Tacconi, ${ }^{29,30}$ X. W. Tang, ${ }^{6}$ Z. C. Tang, ${ }^{6}$ D. Tescaro, ${ }^{24}$ Samuel C. C. Ting, ${ }^{10,16}$ S. M. Ting, ${ }^{10}$ N. Tomassetti ${ }^{32,33}$ J. Torsti, ${ }^{45}$ C. Türkoğlu, ${ }^{2}$ T. Urban, ${ }^{21}$ V. Vagelli, ${ }^{32,33}$ E. Valente, ${ }^{37,38}$ E. Valtonen, ${ }^{45}$ M. Vázquez Acosta, ${ }^{24}$ M. Vecchi,${ }^{39}$ M. Velasco, ${ }^{27}$ J. P. Vialle, ${ }^{3}$ L. Q. Wang ${ }^{41}$ N. H. Wang, ${ }^{41}$ Q. L. Wang, ${ }^{5}$ X. Wang, ${ }^{10}$ X. Q. Wang,${ }^{6,7}$ Z. X. Wang, ${ }^{19}$ C. C. Wei, ${ }^{44, i}$ Z. L. Weng, ${ }^{10}$ K. Whitman, ${ }^{20}$ H. Wu ${ }^{31}$ X. Wu, ${ }^{17}$ R. Q. Xiong, ${ }^{31}$ W. Xu, ${ }^{10}$ Q. Yan, ${ }^{10}$ J. Yang, ${ }^{40}$ M. Yang, ${ }^{6}$ Y. Yang, ${ }^{42}$ H. Yi, ${ }^{31}$ Y. J. Yu, ${ }^{5}$ Z. Q. Yu, ${ }^{6}$ M. Zannoni, ${ }^{29,30}$ S. Zeissler, ${ }^{23}$ C. Zhang, ${ }^{6}$ F. Zhang, ${ }^{6}$ J. Zhang, ${ }^{10, \mathrm{c}}$ J. H. Zhang, ${ }^{31}$ S. W. Zhang, ${ }^{6,7}$ Z. Zhang, ${ }^{10}$ Z. M. Zheng, ${ }^{4}$ H. L. Zhuang, ${ }^{6}$ V. Zhukov, ${ }^{1}$ A. Zichichi, ${ }^{8,9}$ N. Zimmermann, ${ }^{1}$ and P. Zuccon ${ }^{10}$

(AMS Collaboration)

\footnotetext{
${ }^{1}$ I. Physics Institute and JARA-FAME, RWTH Aachen University, D-52056 Aachen, Germany

${ }^{2}$ Department of Physics, Middle East Technical University (METU), 06800 Ankara, Turkey

${ }^{3}$ Laboratoire d'Annecy-le-Vieux de Physique des Particules (LAPP), CNRS/IN2P3 and Université Savoie Mont Blanc, F-74941 Annecy-le-Vieux, France

${ }^{4}$ Beihang University (BUAA), Beijing, 100191, China

${ }^{5}$ Institute of Electrical Engineering (IEE), Chinese Academy of Sciences, Beijing, 100190, China

${ }^{6}$ Institute of High Energy Physics (IHEP), Chinese Academy of Sciences, Beijing, 100049, China

${ }^{7}$ University of Chinese Academy of Sciences (UCAS), Beijing, 100049, China

${ }^{8}$ INFN Sezione di Bologna, I-40126 Bologna, Italy

${ }^{9}$ Università di Bologna, I-40126 Bologna, Italy

${ }^{10}$ Massachusetts Institute of Technology (MIT), Cambridge, Massachusetts 02139, USA

${ }^{11}$ National Central University (NCU), Chung-Li, Tao Yuan, 32054, Taiwan

${ }^{12}$ East-West Center for Space Science, University of Maryland, College Park, Maryland 20742, USA

${ }^{13}$ IPST, University of Maryland, College Park, Maryland 20742, USA

${ }^{14}$ CHEP, Kyungpook National University, 41566 Daegu, Korea

${ }^{15}$ CNR-IROE, I-50125 Firenze, Italy
} 


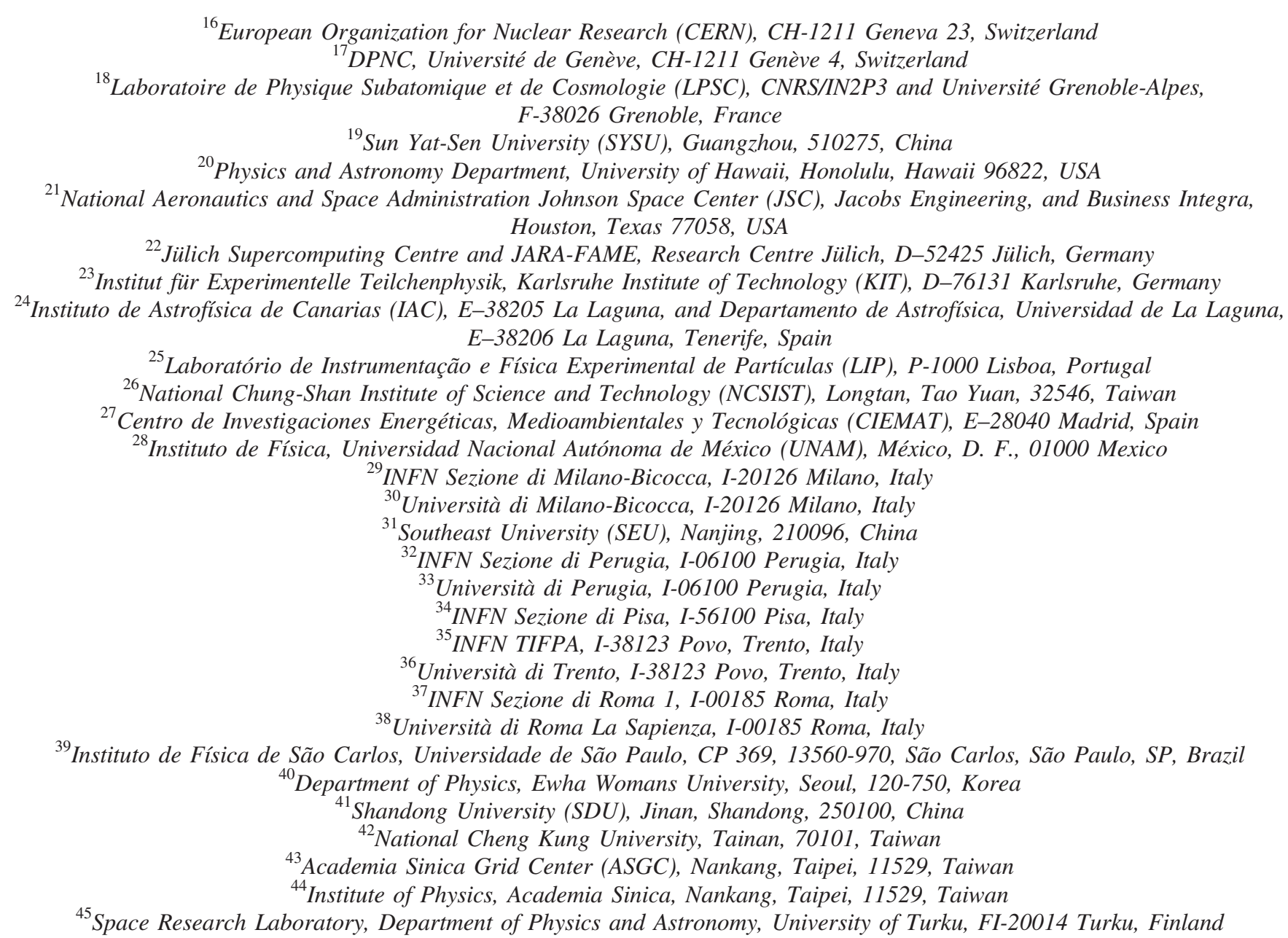

(Received 21 November 2017; revised manuscript received 9 May 2018; published 31 July 2018)

\begin{abstract}
We present the precision measurement from May 2011 to May 2017 (79 Bartels rotations) of the proton fluxes at rigidities from 1 to $60 \mathrm{GV}$ and the helium fluxes from 1.9 to $60 \mathrm{GV}$ based on a total of $1 \times 10^{9}$ events collected with the Alpha Magnetic Spectrometer aboard the International Space Station. This measurement is in solar cycle 24, which has the solar maximum in April 2014. We observed that, below $40 \mathrm{GV}$, the proton flux and the helium flux show nearly identical fine structures in both time and relative amplitude. The amplitudes of the flux structures decrease with increasing rigidity and vanish above $40 \mathrm{GV}$. The amplitudes of the structures are reduced during the time period, which started one year after solar maximum, when the proton and helium fluxes steadily increase. Above $\sim 3 \mathrm{GV}$ the $p / \mathrm{He}$ flux ratio is time independent. We observed that below $\sim 3 \mathrm{GV}$ the ratio has a long-term decrease coinciding with the period during which the fluxes start to rise.
\end{abstract}

DOI: 10.1103/PhysRevLett.121.051101

Cosmic rays entering the heliosphere are subject to diffusion, convection, adiabatic energy losses, and magnetic drift [1]. The temporal evolution of these processes

Published by the American Physical Society under the terms of the Creative Commons Attribution 4.0 International license. Further distribution of this work must maintain attribution to the author(s) and the published article's title, journal citation, and DOI. leads to cosmic ray intensity variation at Earth's orbit around the Sun. These variations correlate with solar activity, which has several cycles [2]. The most significant is the 11-year solar cycle during which the number of sunspots changes from minimum to maximum and then back to a minimum. Another is the 22-year cycle of the Sun's magnetic field polarity, which reverses every 11 years during the maxima of the solar cycle [3]. Cosmic ray spectra may also have temporary reductions due to the 
interactions of cosmic rays with strong disturbances in the magnetic field, especially during solar maxima, that can last from days to months [4-7]. Time correlations at low rigidity among different particle spectra $(p, \mathrm{He})$ due to solar modulation are expected by models of cosmic ray transport based on the Parker equation [1]. This is because the timedependent cosmic ray transport in the heliosphere is rigidity dependent and related to changes in solar activity. Numerous models of the propagation of charged particles in the heliosphere exist that predict different flux variations with time [8-13]. The large acceptance and high precision of the Alpha Magnetic Spectrometer (AMS) allow us to perform accurate measurements of the fluxes as functions of time and energy. This provides unique information to probe the dynamics of solar modulation, to allow the improvement of constraints for dark matter search [14-17], to investigate the processes of galactic cosmic ray propagation $[18,19]$, and to reduce the uncertainties in radiation dose predictions for deep space human exploration $[20,21]$.

The precision, high-energy measurements of the proton and helium fluxes by the AMS have been reported [22,23]. In this Letter, the time evolution of the proton flux from 1 to $60 \mathrm{GV}$ based on $846 \times 10^{6}$ events and the helium flux from 1.9 to $60 \mathrm{GV}$ based on $112 \times 10^{6}$ events are presented. The proton flux and the helium flux are measured for the 79 Bartels rotations from May 2011 to May 2017. For the first time, proton and helium fluxes are simultaneously measured with the same precision instrument for an extended period of time.

Detector.-The layout and description of the AMS detector are presented in Ref. [24]. The key elements used in this measurement are the permanent magnet [25], the silicon tracker [26], and the four planes of time of flight scintillation counters [27]. The AMS also contains a transition radiation detector, a ring imaging Čerenkov detector, an electromagnetic calorimeter, and an array of 16 anticoincidence counters. Proton and helium nuclei traversing the AMS were triggered as described in Refs. [22,23,28] with measured efficiencies of $>94 \%$ up to $60 \mathrm{GV}$. Monte Carlo simulated events were produced using a dedicated program developed by the collaboration based on the GEANT-4.10.1 package [29]. The program simulates electromagnetic and hadronic interactions of particles in the material of the AMS and generates detector responses. The Monte Carlo event samples have sufficient statistics such that they do not contribute to the errors.

Event selection.-The collection time used in this analysis includes only those seconds during which the detector was in normal operating conditions, the AMS was pointing within $40^{\circ}$ of the local zenith, and the International Space Station (ISS) was outside of the South Atlantic Anomaly. In addition, those seconds when the AMS detects solar energetic particles accelerated by the Sun are excluded. Because of the geomagnetic field, the collection time increases with rigidity; it is $1.0-1.4 \times 10^{5} \mathrm{~s}$ at $2 \mathrm{GV}$, $4.2-4.7 \times 10^{5} \mathrm{~s}$ at $5 \mathrm{GV}, 8.8-9.4 \times 10^{5} \mathrm{~s}$ at $10 \mathrm{GV}$, $1.4-1.6 \times 10^{6} \mathrm{~s}$ at $20 \mathrm{GV}$, and, above $30 \mathrm{GV}$, reaches $1.7-1.9 \times 10^{6} \mathrm{~s}$ per Bartels rotation. Proton and helium events were selected as described in Refs. [22,23]. The measured rigidity is required to be greater than a factor of 1.2 times the maximum geomagnetic cutoff within the AMS field of view. The cutoff was calculated by backtracing particles from the top of the AMS out to 50 Earth's radii [30] using the most recent IGRF model [31]. After selection the event samples contain $846 \times 10^{6} Z=+1$ and $112 \times 10^{6} Z=+2$ nuclei each with a purity $>99.8 \%$. The $Z=+1$ sample includes protons and deuterons with rigidity larger than $1.00 \mathrm{GV}$ and $Z=+2$ sample includes ${ }^{3} \mathrm{He}$ and ${ }^{4} \mathrm{He}$ isotopes with rigidity larger than $1.92 \mathrm{GV}$.

Data analysis. - The isotropic flux $\Phi_{i}$ during a Bartels rotation in the $i$ th rigidity bin $\left(R_{i}, R_{i}+\Delta R_{i}\right)$ is given by

$$
\Phi_{i}=\frac{N_{i}}{A_{i} \epsilon_{i} T_{i} \Delta R_{i}},
$$

where, for that Bartels rotation, $N_{i}$ is the number of events corrected for bin-to-bin migration, $A_{i}$ is the effective acceptance, $\epsilon_{i}$ is the trigger efficiency, and $T_{i}$ is the collection time. In this Letter, the proton flux was measured in 45 bins from 1 to $60 \mathrm{GV}$ and the helium flux in 40 bins from 1.9 to $60 \mathrm{GV}$. Above $1.9 \mathrm{GV}$, proton and helium have 40 common rigidity bins with identical bin widths. Bin-tobin migration of events was corrected using the unfolding procedures described in Refs. [22,23] independently for each Bartels rotation for the proton samples and for the helium samples. Extensive studies were made of the systematic errors for each Bartels rotation as described in Refs. $[22,23,28]$. These errors include the uncertainties in the acceptance, due to event reconstruction, selection, and nuclear cross sections, the background contamination, the geomagnetic cutoff factor, the event selection, the unfolding, the rigidity resolution function, and the absolute rigidity scale. These systematic errors are time independent. As an example, to estimate the systematic errors due to uncertainty on the cutoff determination, the nominal geomagnetic cutoff factor of 1.2 was varied from 1.0 to 1.4 and the difference in the resulting fluxes was included in the total systematic errors. The corresponding systematic uncertainties were found to be $2 \%$ at $1 \mathrm{GV}$ for protons and negligible above $2 \mathrm{GV}$ for both protons and helium. As described in Ref. [22], we have also verified that the IGRF model with external nonsymmetric magnetic fields does not introduce observable changes in the flux values. In addition, a time dependent systematic error due to the variations of trigger and reconstruction efficiency for different Bartels rotations was estimated to be $1.5 \%$ for protons at $1 \mathrm{GV}$ and $<1 \%$ at $2 \mathrm{GV},<0.6 \%$ at $10 \mathrm{GV}$, and $<1.2 \%$ at $60 \mathrm{GV}$ for both protons and helium. The total systematic error is obtained by adding in quadrature the 
individual contributions of the time independent systematic errors and the time dependent systematic errors. At $1 \mathrm{GV}$ it is $4.8 \%$ for protons, and it is $<2.5 \%$ above $2 \mathrm{GV}$ for both protons and helium. Most importantly, several independent analyses were performed on the same data sample. The results of those analyses are consistent with those presented in this Letter.

Results.-The measured proton fluxes, helium fluxes, and the $p / \mathrm{He}$ flux ratios for Bartels rotations 2426 to 2506 including statistical errors, time dependent systematic errors, and total systematic errors are tabulated in the Supplemental Material [32], as functions of the rigidity at the top of the AMS detector. Because of the very high statistics, the small time dependent systematic error from trigger and reconstruction efficiency variations for protons and differently for helium are noticeable. As in Ref. [28], contributions to the total systematic error are from the acceptance, the background contamination, the geomagnetic cutoff factor, the event selection, the unfolding, the rigidity resolution function, the absolute rigidity scale, and the time dependent systematic errors. The statistical errors for the ratio are the sum in quadrature of the relative statistical errors of the fluxes multiplied by the ratio. The time dependent systematic errors for the ratio are the sum in quadrature of the relative time dependent systematic errors of the fluxes multiplied by the ratio. The systematic errors from the acceptance for the ratio are added in quadrature. The correlations in the systematic errors from the unfolding and the absolute rigidity scale between the fluxes have been accounted for in calculating the corresponding systematic errors of the ratio. The contributions of the individual sources to the systematic error are added in quadrature to arrive at the total systematic uncertainty on the ratio.

Figure 1 shows the detailed behavior of (a) the proton flux and (b) the helium flux as functions of time and of rigidity from 1 to $10 \mathrm{GV}$ and from 1.9 to $10 \mathrm{GV}$, respectively. Figure SM 1 in the Supplemental Material [32] shows the data over the entire rigidity range up to $60 \mathrm{GV}$. As seen, both the proton and helium spectra exhibit large variations with time at low rigidities which decrease with increasing rigidity. During the period of observation, both fluxes have a minimum in February 2014 and a maximum in February 2017.

The time dependence of the proton and helium fluxes are shown in Fig. 2 for 8 characteristic rigidity bins. As seen, both the proton and helium fluxes have fine time structures each with maxima and minima with boundaries marked by the vertical dashed lines from I to X. The structures in the proton flux and the helium flux are nearly identical in both time and relative amplitude.

In general, the amplitudes of the structures (indicated by the shading) decrease progressively with rigidity. The precision of AMS enables us to observe these structures up to $40 \mathrm{GV}$. The data presented in this Letter provide
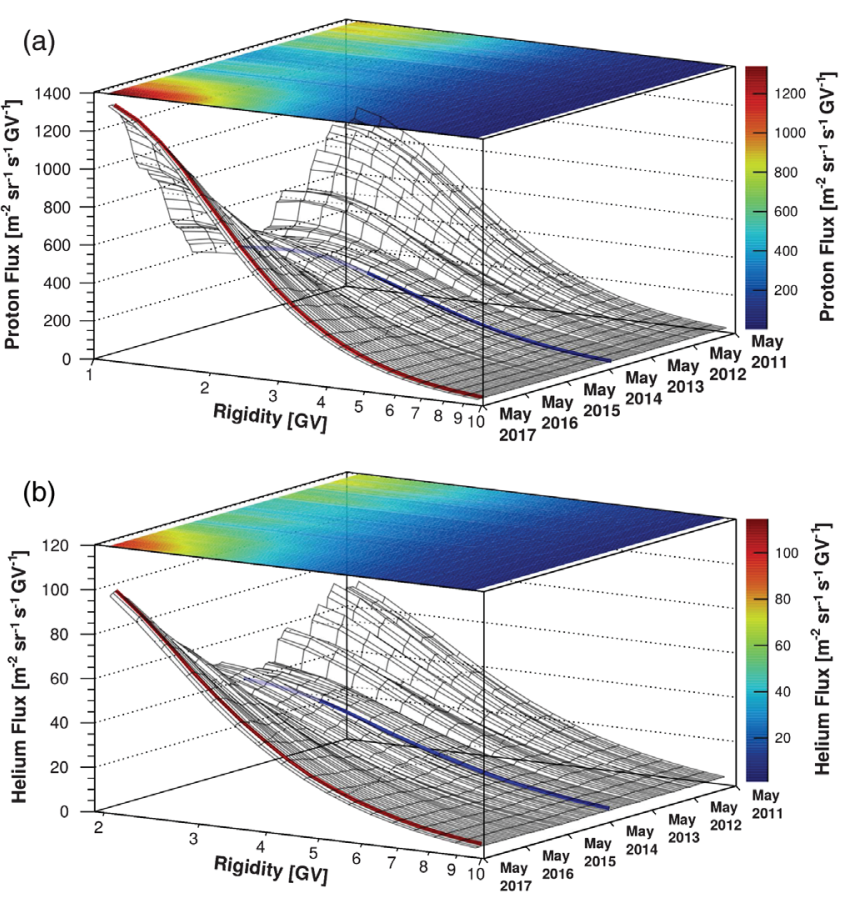

FIG. 1. The three-dimensional detailed behavior of the AMS (a) proton and (b) helium fluxes as functions of rigidity from 1 to $10 \mathrm{GV}$ and from 1.9 to $10 \mathrm{GV}$, respectively, and time. The color code indicates the flux intensity in units of $\left[\mathrm{m}^{2} \cdot \mathrm{sr} \cdot \mathrm{s} \cdot \mathrm{GV}\right]^{-1}$. During the period of observation, both fluxes have a distinct minimum in February 2014 (blue line) and a maximum in February 2017 (red line).

information for detailed studies on time-dependent phenomena like those described in Refs. [34,35].

It is important to note that five of the structures, boundaries I (September 27, 2011), II (March 7, 2012), III (July 20, 2012), IV (May 13, 2013), and VII (March 19, 2015), marked by the red vertical dashed lines in the figure, have also been observed by AMS in the electron flux and the positron flux [33]. As seen, after boundary VII, which is one year after solar maximum (April 2014 for solar cycle $24)$, the amplitudes of the structures are considerably reduced and the proton and helium fluxes steadily increase at rigidities less than $40 \mathrm{GV}$. In addition, the change in long term behavior visible at boundary VII was also observed by AMS in the electron flux and the positron flux.

Figure 3(a) shows the comparison of the proton flux in the kinetic energy per nucleon range 1.19 to $1.40 \mathrm{GeV}$ measured by AMS versus time together with the EPHIN/ SOHO measurement [36]. Figure 3(b) shows the AMS helium flux in the kinetic energy per nucleon range 1.11 to $1.28 \mathrm{GeV} / n$. Figure 3(c) shows the relative variation of the AMS proton flux integrated over $R \geq 6.47 \mathrm{GV}$ as a function of time together with the relative variation of the rate reported by the Oulu, Finland neutron monitor [37]. Figure 3(d) shows the monthly averaged sunspot number during solar cycle 24 with the period of solar magnetic field polarity $(A)$ reversal $[38,39]$. As seen, the data greatly 


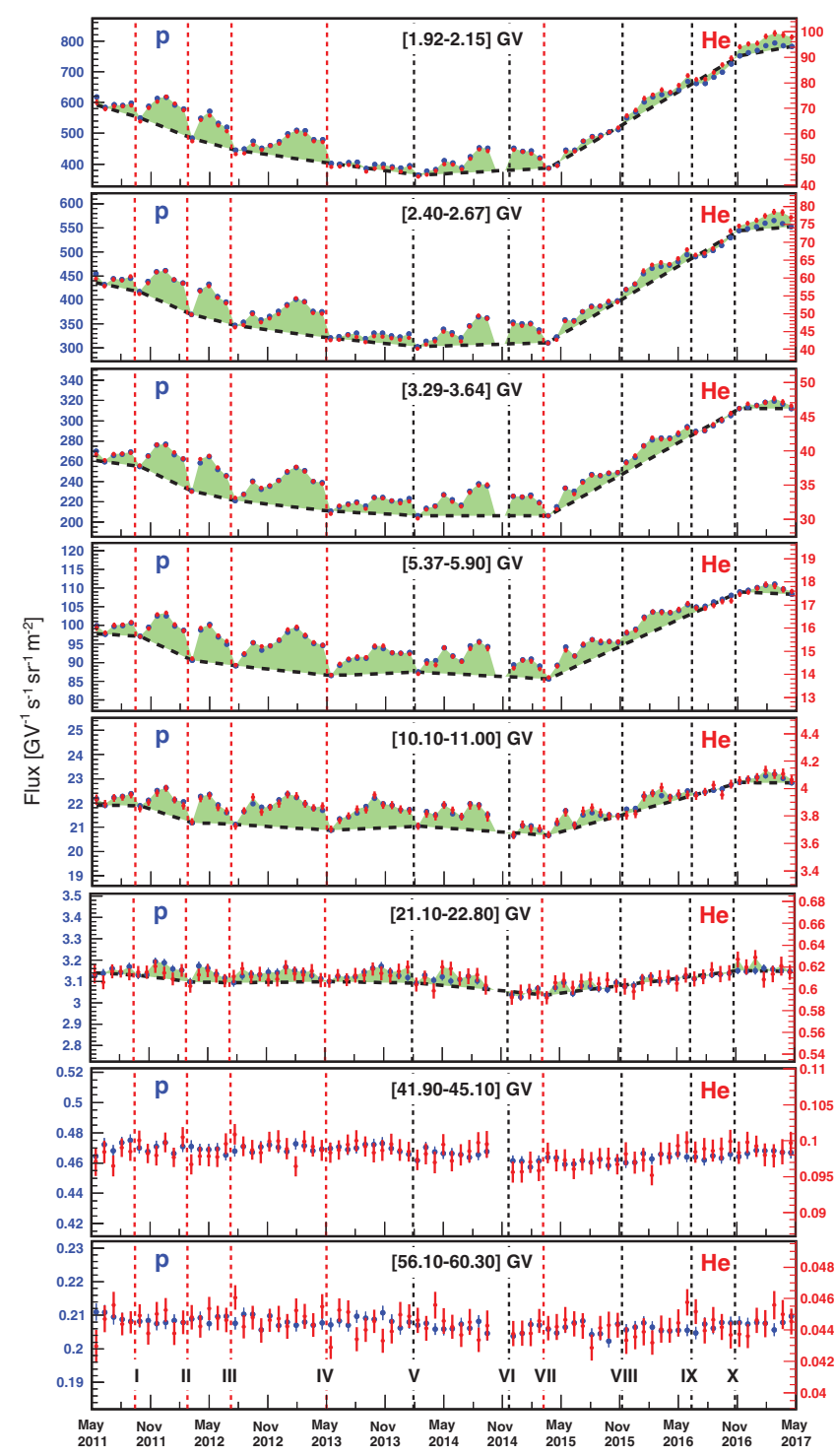

FIG. 2. The AMS proton (blue, left axis) and helium (red, right axis) fluxes as function of time for 8 rigidity bins. The error bars are the quadratic sum of the statistical and time dependent systematic errors. Detailed structures (green shading and dashed lines to guide the eye) are clearly present below $40 \mathrm{GV}$. The vertical dashed lines denote boundaries between these structures at I) September 27, 2011; II) March 7, 2012; III) July 20, 2012; IV) May 13, 2013; V) February 7, 2014; VI) December 1, 2014; VII) March 19, 2015; VIII) November 17, 2015; IX) June 20, 2016; X) November 28, 2016. The red vertical dashed lines denote structures that have also been observed by AMS in the electron flux and the positron flux [33].

improve the accuracy and the sensitivity of the time dependent proton and helium measurements and this provides information for detailed studies of the correlation between sunspot number and the fluxes of protons and helium.

For illustration, Fig. SM 2 in the Supplemental Material [32] shows the relative variation of the AMS proton flux integrated with different minimum rigidities as a function

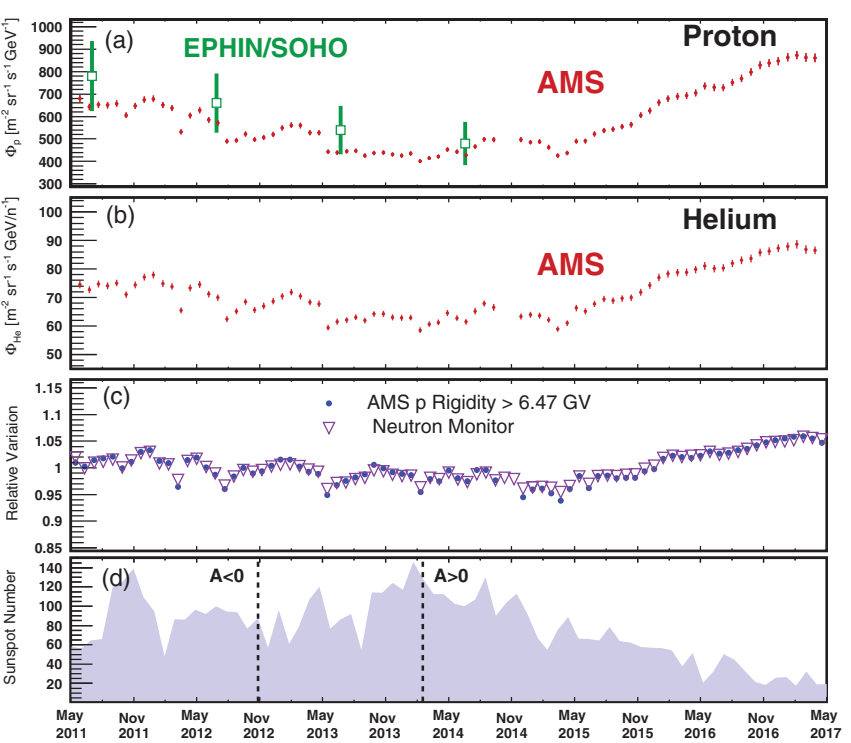

FIG. 3. Comparison of the fine structure time dependence of (a) the AMS proton flux for [1.19-1.40] GeV together with the measurement by EPHIN aboard SOHO for [1.12-1.29] GeV [36], (b) the AMS helium flux for $[1.11-1.28] \mathrm{GeV} / n$, (c) the relative variation of the AMS proton flux integrated over $R \geq$ 6.47 GV as a function of time together with the relative variation of the rate reported by the Oulu, Finland neutron monitor [37], and (d) the monthly averaged sunspot number [38] with the period of solar magnetic field polarity $(A)$ reversal (vertical dashed lines) from $A<0$ to $A>0$, November 2012 to March 2014, of solar cycle 24 [39]. One year after solar maximum, both the $p$ and He fluxes start to rise and, as seen, there is a negative correlation with the sunspot number. AMS data are converted from rigidity $R$ to kinetic energy per nucleon $E_{K}=\left(\sqrt{Z^{2} R^{2}+M^{2}}-M\right) / A$, where $M$ is the proton or the ${ }^{4} \mathrm{He}$ mass. The AMS error bars are the quadratic sum of the statistical and total systematic errors.

of time together with the relative variation of the rate reported by the Oulu, Finland neutron monitor. As seen, the relative variation of this neutron monitor rate matches the AMS proton flux only when the flux is integrated over $R \geq 6.47 \mathrm{GV}$.

Figure 4 shows the AMS $p / \mathrm{He}$ flux ratio, see Supplemental Material [32], as a function of time for 9 rigidity bins. As seen, depending on the rigidity range, the $p /$ He flux ratio shows two different behaviors in time. Above $\sim 3 \mathrm{GV}$ the ratio is time independent. Below $\sim 3 \mathrm{GV}$ the ratio has a long-term time dependence. To assess the transition between these two behaviors, we performed a fit of the $p / \mathrm{He}$ flux ratio $r_{i}$ for each rigidity bin $i$ as a function of time $t$, with

$$
r_{i}(t)= \begin{cases}a_{i} & t<t_{i} \\ a_{i}+b_{i}\left(t-t_{i}\right) & t \geq t_{i},\end{cases}
$$

where $a_{i}$ is the average $p / \mathrm{He}$ flux ratio from May 2011 to $t_{i}, t_{i}$ is the time when the $p /$ He flux ratio deviates from the average $a_{i}$, and $b_{i}$ is the slope of the time variation. Above 


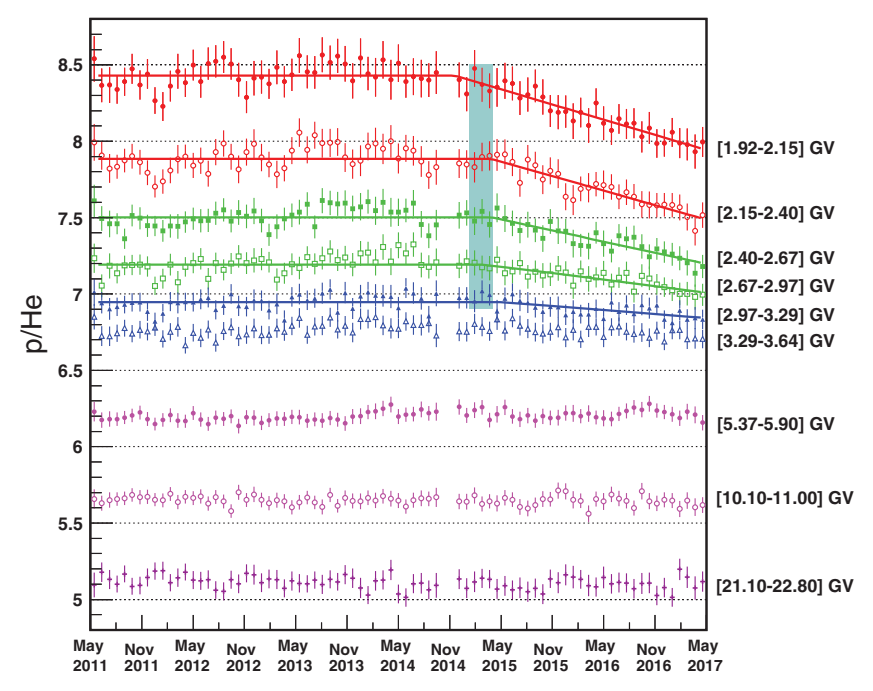

FIG. 4. The AMS $p / \mathrm{He}$ flux ratio as function of time for 9 characteristic rigidity bins. The errors are the quadratic sum of the statistical and time dependent systematic errors. The solid lines are the best fit of Eq. (2) for the first 5 rigidity bins from [1.92-2.15] GV to [2.97-3.29] GV. The vertical band (February $28,2015 \pm 42$ days) is the average of the best fit values of $t_{i}$ for these rigidity bins.

$3.29 \mathrm{GV}$, the $p / \mathrm{He}$ flux ratio is consistent with a constant value at the $95 \%$ confidence level. This shows the universality of the solar modulation of cosmic ray nuclei at relativistic rigidities. Below $3.29 \mathrm{GV}$, the observed $p / \mathrm{He}$ flux ratio is steadily decreasing with time after $t_{i}$. In the first five rigidity bins, the best fit values of $t_{i}$ are in agreement within each other. Their average value is equal to February 28, $2015 \pm 42$ days, consistent with boundary VII of Fig. 2, after which the proton and helium fluxes start to increase. This last observation shows a new and important feature regarding the propagation of lower energy cosmic rays in the heliosphere. Before this Letter, several effects had been proposed that lead to a time dependence of the $p / \mathrm{He}$ flux ratio at low rigidities, such as velocity dependence of the diffusion tensor, differences in the interstellar spectra of $p$ and $\mathrm{He}$, and the ${ }^{3} \mathrm{He}$ and ${ }^{4} \mathrm{He}$ isotopic composition [8-13,28,40,41]. The precision of the AMS data provides information for the development of refined solar modulation models.

In conclusion, the precision proton flux and the helium flux observed by AMS have fine time structures nearly identical in both time and relative amplitude. The amplitudes of the flux structures decrease with increasing rigidity and vanish above $40 \mathrm{GV}$. The amplitudes of the structures are reduced during the time period, which started one year after solar maximum, when the proton and helium fluxes steadily increase. In addition, above $\sim 3 \mathrm{GV}$ the $p / \mathrm{He}$ flux ratio is time independent. Below $\sim 3 \mathrm{GV}$ the ratio has a long-term decrease coinciding with the period during which the fluxes start to rise.

We thank former NASA Administrator Daniel S. Goldin for his dedication to the legacy of the ISS as a scientific laboratory and his decision for NASA to fly the AMS as a DOE payload. We also acknowledge the continuous support of the NASA leadership including Charles Bolden and William H. Gerstenmaier and of the JSC and MSFC flight control teams which has allowed AMS to operate optimally on the ISS for over six years. We are grateful for the support of Jim Siegrist and his staff of the DOE including resources from the National Energy Research Scientific Computing Center under Contract No. DE-AC02-05CH11231 and the Argonne Leadership Computing Facility under Contract No. DE-AC02-06CH11357. We also acknowledge the continuous support from MIT and its School of Science, Michael Sipser, Marc Kastner, Ernest Moniz, Richard Milner, and Boleslaw Wyslouch. We are grateful for the support of Edward Semones and his staff of the NASA Johnson Space Center including resources from Wyle Laboratories Grant No. 2014/T72497. Research supported by São Paulo Research Foundation (FAPESP) Grants No. 2014/19149-7, No. 2015/50378-5, and No. 2016/ 10222-9, Brazil; CAS, NSFC, MOST, the provincial governments of Shandong, Jiangsu, Guangdong, and the China Scholarship Council, China; Action H2020 MSCAIF-2015 under Grant No. 707543-MAtISSE, European Union; the Finnish Funding Agency for Innovation (Tekes) Grants No. 40361/01 and No. 40518/03 and the Academy of Finland Grant No. 258963, Finland; CNRS/ IN2P3, CNES, Enigmass, and the ANR, France; Pascale Ehrenfreund, DLR under Grant No. 50001403 and JARAHPC under Project No. JARA0052, Germany; INFN and ASI under ASI-INFN Agreements No. 2013-002-R.0 and No. 2014-037-R.0, Italy; CHEP and NRF under Grants No. NRF-2009-0080142 and No. NRF-2012-010226 at Kyungpook National University and No. NRF-2013004883 at Ewha Womans University, Korea; the Consejo Nacional de Ciencia y Tecnología and UNAM, Mexico; FCT under Grant No. PTDC/FIS/122567/2010, Portugal; CIEMAT, IAC, CDTI, and SEIDI-MINECO under Grants No. ESP2015-71662-C2-(1-P/2-P), No. SEV-2015-0548, No. MDM-2015-0509, and No. RyC-2013-14660, Spain; the Swiss National Science Foundation (SNSF), federal and cantonal authorities, Switzerland; Academia Sinica and the Ministry of Science and Technology (MOST) under Grants No. 103-2112-M-006-018-MY3, No. 105-2112-M-001-003, and No. CDA-105-M06, former Presidents of Academia Sinica Yuan-Tseh Lee and Chi-Huey Wong and former Ministers of MOST Maw-Kuen Wu and Luo-Chuan Lee, Taiwan; the Turkish Atomic Energy Authority under Grant No. 2017TEAK(CERN)A5.H6.F2-15, Turkey; and NSF Grants No. 14255202 and No. 1551980, and NASA NESSF Grant No. HELIO15F-0005, USA. We gratefully acknowledge the strong support from CERN including RolfDieter Heuer and Fabiola Gianotti, from the CERN IT department and Bernd Panzer-Steindel, and from the European Space Agency including Johann-Dietrich Wörner and Simonetta Di Pippo. We are grateful for 
important physics discussions with Fiorenza Donato, Jonathan Ellis, Jonathan Feng, Igor Moskalenko, Marius Potgieter, Michael Salamon, Subir Sarkar, Joachim Trümper, Michael S. Turner, and Steven Weinberg.

${ }^{a}$ Also at ASI, I-00133 Roma, Italy.

${ }^{\mathrm{b}}$ Also at ASI Space Science Data Center (SSDC), I-00133 Roma, Italy; Present address: University of Sassari, I-07100 Sassari, Italy.

${ }^{c}$ Also at Wuhan University, Wuhan, 430072, China.

${ }^{\mathrm{d}}$ Also at Sun Yat-Sen University (SYSU), Guangzhou, 510275, China.

${ }^{\mathrm{e}}$ Also at Huazhong University of Science and Technology (HUST), Wuhan, 430074, China.

${ }^{\mathrm{f}}$ Also at ASI Space Science Data Center (SSDC), I-00133 Roma, Italy.

${ }^{\mathrm{g}}$ Also at ASI Space Science Data Center (SSDC), I-00133 Roma, Italy; Present address: INFN Sezione di Trieste, I-34149, Trieste, Italy.

${ }^{\mathrm{h}}$ Also at Nankai University, Tianjin 300071, China.

${ }^{\mathrm{i}}$ Also at Institute of Theoretial Physics, Chinese Academy of Sciences, Beijing, 100190, China.

[1] E. N. Parker, Planet. Space Sci. 13, 9 (1965).

[2] M. S. Potgieter, Living Rev. Solar Phys. 10, 3 (2013).

[3] D. H. Hathaway, Living Rev. Solar Phys. 12, 4 (2015).

[4] M. Zhang, Adv. Space Res. 32, 603 (2003).

[5] H. V. Cane, Space Sci. Rev. 93, 55 (2000).

[6] M. S. Potgieter, J. A. Le Roux, L. F. Burlaga, and F. B. McDonald, Astrophys. J. 403, 760 (1993).

[7] G. Wibberenz, I. G. Richardson, and H. V. Cane, J. Geophys. Res. 107, 1353 (2002).

[8] I. Cholis, D. Hooper, and T. Linden, Phys. Rev. D 93, 043016 (2016).

[9] C. Corti, V. Bindi, C. Consolandi, and K. Whitman, Astrophys. J. 829, 8 (2016).

[10] M. J. Boschini, S. Della Torre, M. Gervasi, G. La Vacca, and P. G. Rancoita, Adv. Space Res., DOI: 10.1016/ j.asr.2017.04.017 (2017).

[11] J. Gieseler, B. Heber, and K. Herbst, J. Geophys. Res. Space Phys. 122, 10964 (2017).

[12] N. Tomassetti, M. Orcinha, F. Barão, and B. Bertucci, Astrophys. J. Lett. 849, L32 (2017).

[13] E. E. Vos and M. S. Potgieter, Astrophys. J. 815, 119 (2015).

[14] N. Fornengo, L. Maccione, and A. Vittino, J. Cosmol. Astropart. Phys. 9 (2013) 031.

[15] N. Fornengo, L. Maccione, and A. Vittino, J. Cosmol. Astropart. Phys. 4 (2014) 003.

[16] M. Cirelli, D. Gaggero, G. Giesen, M. Taoso, and A. Urbano, J. Cosmol. Astropart. Phys. 12 (2014) 045.

[17] Q. Yuan and X.-J. Bi, J. Cosmol. Astropart. Phys. 3 (2015) 033.

[18] M. J. Boschini et al., Astrophys. J. 840, 115 (2017).

[19] N. Tomassetti, Phys. Rev. D 96, 103005 (2017).

[20] P. M. O’Neill, IEEE Trans. Nucl. Sci. 57, 3148 (2010).

[21] L. W. Townsend, F. A. Cucinotta, J. W. Wilson, J. L. Shinn, and G. Badhwar, Adv. Space Res. 14, 853 (1994).

[22] M. Aguilar et al., Phys. Rev. Lett. 114, 171103 (2015).

[23] M. Aguilar et al., Phys. Rev. Lett. 119, 251101 (2017).
[24] A. Kounine, Int. J. Mod. Phys. E 21, 1230005 (2012); S. Rosier-Lees, Proceedings of Astroparticle Physics TEVPA/ IDM, Amsterdam, 2014 (unpublished); S. Ting, Nucl. Phys. B, Proc. Suppl. 243-244, 12 (2013); S.-C. Lee, Proceedings of the 20th International Conference on Supersymmetry and Unification of Fundamental Interactions (SUSY Beijing, 2012) (unpublished); M. Aguilar, Proceedings of the $X L$ International Meeting on Fundamental Physics, Centro de Ciencias de Benasque Pedro Pascual, 2012 (unpublished); S. Schael, Proceedings of the 10th Symposium on Sources and Detection of Dark Matter and Dark Energy in the Universe, Los Angeles, 2012 (unpublished); B. Bertucci, Proc. Sci., EPS-HEP (2011) 67; M. Incagli, AIP Conf. Proc. 1223, 43 (2010); R. Battiston, Nucl. Instrum. Methods Phys. Res., Sect. A 588, 227 (2008).

[25] K. Lübelsmeyer et al., Nucl. Instrum. Methods Phys. Res., Sect. A 654, 639 (2011).

[26] B. Alpat et al., Nucl. Instrum. Methods Phys. Res., Sect. A 613, 207 (2010).

[27] V. Bindi et al., Nucl. Instrum. Methods Phys. Res., Sect. A 743, 22 (2014).

[28] M. Aguilar et al., Phys. Rev. Lett. 115, 211101 (2015).

[29] J. Allison et al., Nucl. Instrum. Methods Phys. Res., Sect. A 835, 186 (2016); IEEE Trans. Nucl. Sci. 53, 270 (2006); S. Agostinelli et al., Nucl. Instrum. Methods Phys. Res., Sect. A 506, 250 (2003).

[30] J. Alcaraz et al., Phys. Lett. B 484, 10 (2000).

[31] C. C. Finlay et al., Geophys. J. Int. 183, 1216 (2010); E. Thébault et al., Earth Planets Space 67, 79 (2015).

[32] See Supplemental Material at http://link.aps.org/ supplemental/10.1103/PhysRevLett.121.051101 for figures of the $p$ and He fluxes and of the integrated $p$ flux compared with neutron monitor counts and for the tabulated proton flux, helium flux, and the $p /$ He flux ratio for Bartels rotations 2426 to 2506. Data for Bartels rotation 2472 and 2473 are not provided as AMS was performing detector studies over that interval. The data can also be downloaded in different formats from the ASI cosmic-ray database at https://tools.asdc.asi.it/ CosmicRays/.

[33] M. Aguilar et al., following Letter, Phys. Rev. Lett. 121, 051102 (2018).

[34] L. F. Burlaga, J. Perko, and J. Pirraglia, Astrophys. J. 407, 347 (1993).

[35] G. Newkirk Jr., A. J. Hundhausen, and V. Pizzo, J. Geophys. Res. 86, 5387 (1981).

[36] P. Kühl, R. Gómez-Herrero, and B. Heber, Sol. Phys. 291, 965 (2016).

[37] We acknowledge the NMDB database at http://www.nmdb .eu, founded under the European Union's FP7 program, Contract No. 213007, for providing data.

[38] Sunspot data from the World Data Center SILSO, Royal Observatory of Belgium, Brussels at http://www.sidc.be/ silso/datafiles.

[39] X. Sun, J. T. Hoeksema, Y. Liu, and J. Zhao, Astrophys. J. 798, 114 (2015).

[40] G. Gloeckler and J. R. Jokipii, Phys. Rev. Lett. 17, 203 (1966).

[41] A. C. Cummings, E. C. Stone, B. C. Heikkila, N. Lal, W. R. Webber, G. Jóhannesson, and I. V. Moskalenko, Astrophys. J. 831, 18 (2016). 